\title{
Silicate-free growth of high-quality ultrathin cerium oxide films on $\mathrm{Si}(111)$
}

\author{
Jan Ingo Flege, ${ }^{1, *}$ Björn Kaemena, ${ }^{1}$ Sebastian Gevers, ${ }^{2}$ Florian Bertram, ${ }^{3}$ Torsten Wilkens, ${ }^{1}$ Daniel Bruns, ${ }^{2}$ Jan Bätjer, ${ }^{3}$ \\ Thomas Schmidt, ${ }^{1}$ Joachim Wollschläger, ${ }^{2}$ and Jens Falta ${ }^{1}$ \\ ${ }^{1}$ Institute of Solid State Physics, University of Bremen, Otto-Hahn-Allee 1, D-28359 Bremen, Germany \\ ${ }^{2}$ Department of Physics, University of Osnabrück, Barbarastraße 7, D-49069 Osnabrück, Germany \\ ${ }^{3}$ Hamburger Synchrotronstrahlungslabor am Deutschen Elektronensynchrotron, Notkestraße 85, D-22607 Hamburg, Germany
}

(Received 20 October 2011; revised manuscript received 16 November 2011; published 2 December 2011)

\begin{abstract}
Ultrathin $\mathrm{Ce}_{2} \mathrm{O}_{3}$ layers have been grown on $\mathrm{Si}(111)$ by reactive metal deposition in an oxygen background and characterized by $\mathrm{x}$-ray standing waves, $\mathrm{x}$-ray diffraction, $\mathrm{x}$-ray photoelectron spectroscopy, and low-energy electron diffraction to elucidate and quantify both atomic structure and chemical composition. It is demonstrated that highly ordered, mostly $B$-oriented, epitaxial ceria films can be achieved by preadsorption of a monolayer of atomic chlorine, effectively passivating the substrate and thereby suppressing cerium silicate and silicon oxide formation at the interface.
\end{abstract}

DOI: 10.1103/PhysRevB.84.235418

PACS number(s): 68.49.Uv, 61.05.cp, 68.55.-a, 81.05.Je

\section{INTRODUCTION}

The realization of high-quality, epitaxial thin oxide films on silicon is a long-standing goal in semiconductor technology and may ultimately be regarded as a requirement to maintain the exponential increase in integration density witnessed in microelectronics over the past decades, which is commonly referred to as Moore's law. Generally, further downscaling of field-effect transistor (FET) devices is strongly impeded by exponentially increasing leakage currents in the gate oxide region due to the tunneling effect through thin-film insulators. To overcome the associated inevitable power loss, the gate oxide thickness has to be increased at constant or even enlarged capacitance density. This conservation of capacitance is crucial for correct device operation since it delimits the accessible range of charge carrier density in the inversion layer and thus the achievable source-drain current at given gate voltage. ${ }^{1}$ Hence, this goal may only be achieved using insulating materials that exhibit a higher dielectric constant $k$ than, e.g., $\mathrm{SiO}_{2}$, which has been the oxide of choice in conventional FETs due to its otherwise favorable electronic properties.

Among other applications, e.g., in catalysis, ${ }^{2}$ cerium oxide is very promising for the realization of novel "high- $k$ " dielectrics due to its predicted high thermodynamic stability in contact with silicon. ${ }^{3}$ In contrast to other binary oxides e.g., $\mathrm{HfO}_{2}$, that may only be deposited as amorphous oxide films, it provides two crystallographic phases that are promising for epitaxial growth on silicon, offering the possibility for interface engineering ${ }^{4}$ by molecular beam epitaxy (MBE), which is expected to result in reduced leakage currents. While both the fluorite $\left(\mathrm{CeO}_{2}\right)$ and the cubic bixbyite phase $\left(\mathrm{Ce}_{2} \mathrm{O}_{3}\right)$ may generally be used as high- $k$ dielectrics, the very small lattice mismatch of $\mathrm{CeO}_{2}$ to silicon $(\sim-0.35 \%)^{5}$ and its even higher $k$-value of more than 50 (Refs. 6 and 7) make this form more attractive. However, growth ${ }^{5,8,9}$ of high-quality ultrathin films, which is crucial for optimized integration, has so far been considerably impeded by Ce-promoted Si oxidation at the interface, resulting in subsequent silicon oxide and cerium silicate formation. ${ }^{10-12}$ Yet, despite its higher lattice mismatch of about $2.7 \%$ using two silicon lattice constants as reference, ${ }^{13}$ cubic $\mathrm{Ce}_{2} \mathrm{O}_{3}$ is generally believed to be well suited as a buffer layer for sequential $\mathrm{CeO}_{2}$ deposition due to its lower oxidation state, potentially avoiding the concomitant evolution of $\mathrm{SiO}_{x}$ and $\mathrm{CeSi}_{x} \mathrm{O}_{y}$ species. These low- $k$ defect phases give rise to significant interfacial charge trapping while lowering the dielectric constant of the film and increasing the effective oxide thickness (EOT) of the metal insulator semiconductor field-effect transistor (MISFET) structure. ${ }^{6}$ Consequently, previous attempts in direct growth of $\mathrm{CeO}_{2}$ on $\mathrm{Si}(111)$ have resulted in worse MISFET performance with respect to saturation drain current and subthreshold behavior, effectively making intentionally postoxidized stack structures with a considerably thickened amorphous interface layer still more attractive. ${ }^{6}$ Hence, fabrication of a defect-free, high- $k$, epitaxial interface layer remains of crucial importance for the realization of novel MISFET structures with further decreased EOTs.

Conceivable routes to overcome the challenges presented by the high reactivity at the oxide-silicon interface could, in principle, range from attempts to influence the growth kinetics by varying the ceria growth rate, oxygen partial pressure, and substrate temperature ${ }^{14}$ to approaches that incorporate surface active agents, e.g., hydrogen, ${ }^{6,14,15}$ to stabilize the interface before reactive cerium deposition in an oxygen background. The latter option, unfortunately, carries the disadvantage that the relatively low desorption temperature of hydrogen from silicon [about $450^{\circ} \mathrm{C}$ (Ref. 16)] imposes an upper limit on the growth temperature range, which directly affects the level of crystalline quality of the film that can be obtained.

In this contribution, we present an extensive study of cerium oxide films epitaxially grown on chlorine-passivated $\mathrm{Si}(111)$ by reactive MBE under ultrahigh-vacuum conditions. After introducing the cerium oxide-chlorine-silicon system by supplemental methods, we focus on the application of the $\mathrm{x}$-ray standing-wave (XSW) technique for ultrathin oxide film analysis and the role of the growth modifier $\mathrm{Cl}$ (see Sec. III D). Chlorine, whose $(1 \times 1)$-reconstructed adsorbate structure on $\mathrm{Si}(111)$ is well known from several studies, ${ }^{17-19}$ including the XSW technique, ${ }^{20,21}$ serves multiple purposes in this context. First, the $\mathrm{Cl} / \mathrm{Si}(111)-(1 \times 1)$ system is known to be very stable against oxidation and contamination under near-UHV 
conditions, ${ }^{18}$ which is exploited in the initial growth phase where oxidation of the silicon surface would be most disruptive for subsequent cerium oxide film formation. Second, the desorption temperature of chlorine from $\mathrm{Si}(111)$ amounts to about $680^{\circ} \mathrm{C},{ }^{22}$ allowing for considerably increased growth temperatures as compared to hydrogen preadsorption. Lastly, again in contrast to hydrogen, chlorine can also easily be traced by spectroscopic techniques, enabling the determination of the chlorine binding site and elucidating its role in the cerium oxide growth mechanism.

\section{EXPERIMENTAL}

All investigations presented here were performed at the Hamburg Synchrotron Radiation Laboratory (HASYLAB), which is located at the Deutsches Elektronensynchrotron (DESY) in Hamburg, Germany. At the undulator beam line BW1, the samples were prepared and characterized in situ under UHV conditions employing XSW, x-ray photoemission spectroscopy (XPS), and low-energy electron diffraction (LEED). The XSW experiments were conducted in a nondispersive setup employing a water-cooled $\mathrm{Si}(111)$ double-crystal monochromator including an asymmetrically cut second crystal for improved phase contrast, using primary photon energies between 2.4 and $5.9 \mathrm{keV}$. The $\mathrm{x}$-ray reflectometry (XRR) and (grazing-incidence) $\mathrm{x}$-ray diffraction [(GI)XRD] investigations were conducted ex situ at a photon energy of $10 \mathrm{keV}$ provided by the wiggler beam line BW2.

In the case of the XSW measurements, the preparation of the samples started from polished, RCA-cleaned $\mathrm{Si}(111)$ crystals while in all other experiments also conventional $\mathrm{Si}(111)$ wafers were used. After degassing the samples for at least $12 \mathrm{~h}$ at a temperature of $630^{\circ} \mathrm{C}$, the samples were annealed at a temperature of $880^{\circ} \mathrm{C}$ for the RCA-cleaned samples and flashed to temperatures of $1200^{\circ} \mathrm{C}$ for the wafers, respectively, to remove the protective silicon oxide layer. The resulting $(7 \times 7)$ reconstruction was monitored by LEED. [For a representative diffraction pattern, see Fig. 1(a)].

For the reference samples, cerium oxide was grown by reactive $\mathrm{MBE}$ on the clean $\operatorname{Si}(111)-(7 \times 7)$ surface at a substrate temperature of $500^{\circ} \mathrm{C}$, employing an electron beam evaporator for deposition of metallic $\mathrm{Ce}$ at preset oxygen background pressures between $1 \times 10^{-7}$ and $1 \times 10^{-6}$ mbar. Typical cerium oxide growth rates amounted to $2-3 \AA / \mathrm{min}$ as determined from XRR.

In the modified growth recipe, first, the $\mathrm{Si}(111)$ samples were terminated by 1 monolayer (ML) of atomic $\mathrm{Cl}$ by $\mathrm{Cl}_{2}$ saturation exposure from a $\mathrm{AgCl}$ electrochemical source $\mathrm{e}^{21,23}$ at a substrate temperature of $600{ }^{\circ} \mathrm{C}$ (cf. Ref. 21). The complete removal of the dimers and stacking faults of the $(7 \times 7)$ reconstruction results in a threefold-symmetric, sharp $(1 \times 1)$ LEED pattern, which, depending on the electron kinetic energy, exhibits an entire absence of either of the two sets of three symmetrically equivalent first-order spots [Fig. 1(b)]. In the next step, cerium oxide films were grown by reactive MBE at otherwise unchanged conditions, i.e., applying the same substrate temperature, cerium deposition rate, and oxygen partial pressure.

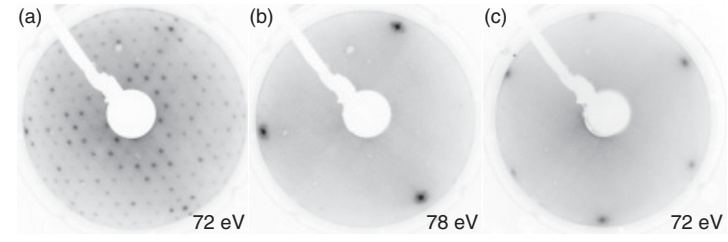

FIG. 1. Low-energy electron diffraction patterns recorded for (a) clean $\mathrm{Si}(111)-(7 \times 7)$, (b) $\mathrm{Cl} / \mathrm{Si}(111)-(1 \times 1)$, and (c) cerium oxide reactively grown on $\mathrm{Cl} / \mathrm{Si}(111)-(1 \times 1)$ in an $\mathrm{O}_{2}$ background of $5 \times 10^{-7}$ mbar.

\section{RESULTS AND DISCUSSION}

In the following sections, we present our experimental results for cerium oxide growth on Cl-passivated silicon substrates and compare these with reference samples prepared without $\mathrm{Cl}$ preadsorption. Using XPS (Sec. III A), we analyze the film morphology, the oxidation state, and its dependence on oxygen partial pressure for film thicknesses up to a few nanometers. Furthermore, surface crystallinity is assessed by qualitative LEED for few-atomic-layer-thin cerium deposits (Sec. III B). In addition, we also report our findings for thicker deposits based on XRR and XRD regarding lattice constants, film orientation, film and interface morphology, and structure (Sec. III C). Finally, using XSW we characterize the atomic structure of ultrathin films and reveal the role of the adsorbed $\mathrm{Cl}$ in cerium oxide growth (Sec. III D).

\section{A. X-ray photoelectron spectroscopy}

We start off our discussion by comparing the chemistry of cerium oxide films grown on the clean $\operatorname{Si}(111)-(7 \times 7)$ surface and chlorine-passivated $\mathrm{Si}(111)$ by reactive $\mathrm{MBE}$ at substrate temperatures of $500^{\circ} \mathrm{C}$ and preset oxygen background pressures. For improved readability, we use a shorthand notation for the preparation conditions as follows: Oxide deposition at $1 \times 10^{-7}$ mbar and $5 \times 10^{-7}$ mbar on substrates with and without chlorine passivation will be labeled by " $(1-\mathrm{Cl})$," “(5-Cl)," “(1)," and "(5)," respectively. Furthermore, when comparing XRD results obtained at ambient conditions, we add respective labels for capped and uncapped samples in the figure captions.

In Fig. 2, representative Ce $3 d$ photoemission spectra are presented for an incident photon energy of $2.4 \mathrm{keV}$, from which the Ce oxidation states in the films can be inferred. Qualitatively, all spectra look very similar, regardless of $\mathrm{Cl}$ preadsorption [(1) vs (1-Cl)] and oxygen partial pressure [(1-Cl) vs (5-Cl)]. Quantitatively, all spectra can consistently be reproduced by fitting the pronounced double peaks to the well-established components $\left(u_{0}, u^{\prime}, v_{0}, v^{\prime}\right)$ associated with the hybridized final state in $\mathrm{Ce}_{2} \mathrm{O}_{3}$ (Ref. 24) using Voigt line shapes and a Tougaard background. Furthermore, we have introduced additional peaks $\left(\mathrm{EL}_{0}, \mathrm{EL}^{\prime}\right)$ that accompany every single component and that we attribute to energy-loss features, e.g., plasmons, due to their constant shift to higher binding energies. These findings indicate the sole formation of $\mathrm{Ce}_{2} \mathrm{O}_{3},{ }^{25}$ i.e., $\mathrm{Ce}^{3+}$, under the present preparation conditions. Since the spectra qualitatively do not exhibit any visible change if the photoelectron kinetic energy is increased by about $1 \mathrm{keV}$ to $\sim 2.5 \mathrm{keV}$, which should allow to probe most (if not all) of 


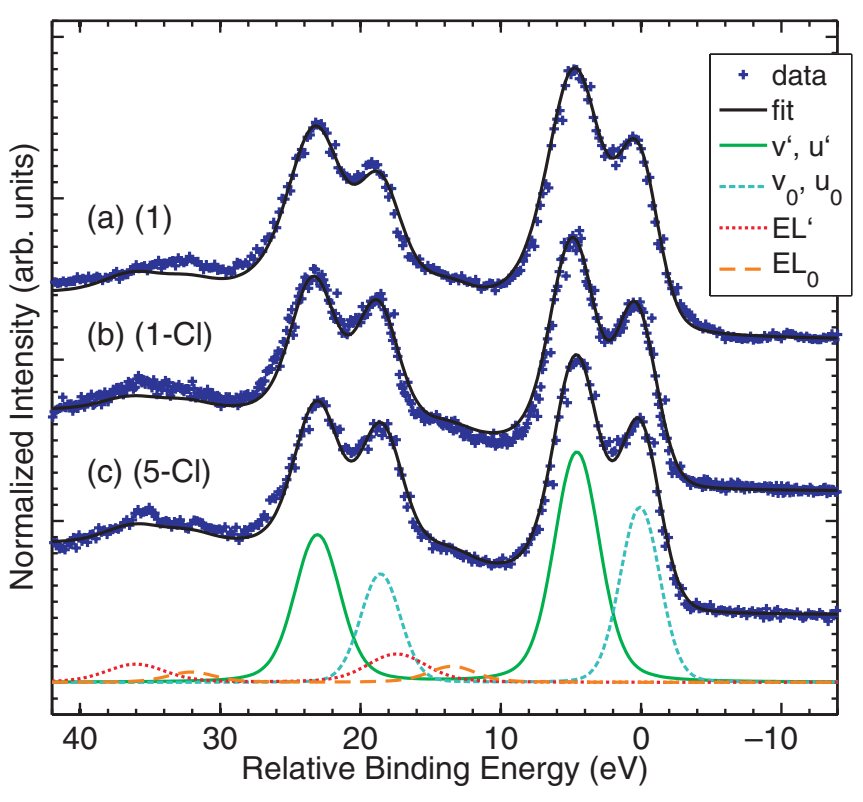

FIG. 2. (Color online) Ce $3 d$ XPS data obtained after cerium oxide growth at a substrate temperature of $500^{\circ} \mathrm{C}$ on (a) clean $\mathrm{Si}(111)$ $(7 \times 7)$ and $1 \times 10^{-7}$ mbar $_{2}$ partial pressure, (1); (b) Cl-passivated $\mathrm{Si}(111)$ and $1 \times 10^{-7}$ mbar $\mathrm{O}_{2}$ partial pressure, (1-Cl); and (c) $\mathrm{Cl}-$ passivated $\mathrm{Si}(111)$ and $5 \times 10^{-7} \mathrm{mbar}_{2}$ partial pressure, $(5-\mathrm{Cl})$. Film thicknesses are a few nanometers in all cases.

the few-nanometer thin film, we may conclude that the film is chemically uniform.

Information regarding the lateral film morphology can be deduced from the variation of the integral $\mathrm{Si} 1 s$ intensity observed in XPS (Fig. 3) with oxide thickness. After the growth of a $-\mathrm{nm}$ thin film using recipe $(1-\mathrm{Cl})$ and using a primary photon energy of $2.4 \mathrm{keV}$, the peak intensity has essentially dropped to zero, proving the formation of a continuous $\mathrm{Ce}_{2} \mathrm{O}_{3}$ film.

Whereas the chemical properties of the films apparently do not change for oxygen partial pressures up to $5 \times$ $10^{-7}$ mbar and film thicknesses in the few-nanometer range, $\mathrm{Cl}$

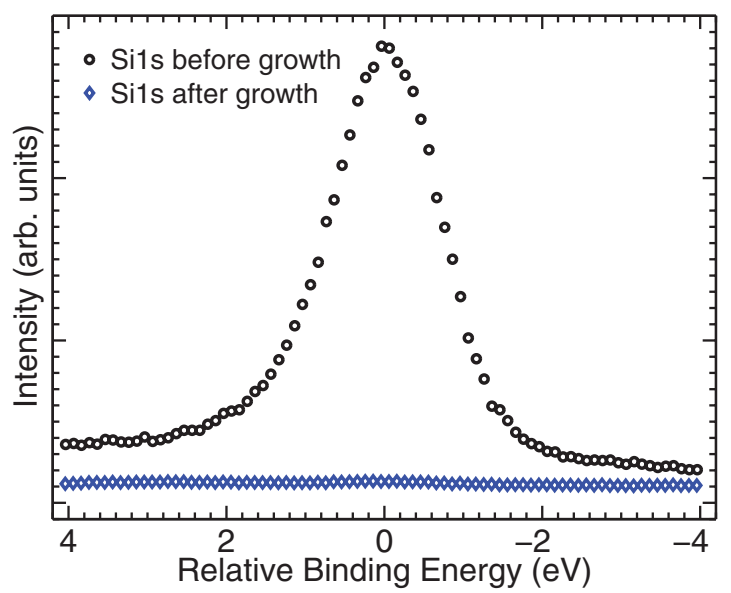

FIG. 3. (Color online) Si $1 s$ XPS data obtained (a) before and (b) after growth of a $2.5-\mathrm{nm}$ thin cerium oxide film at $500^{\circ} \mathrm{C}$ substrate temperature and $1 \times 10^{-7}$ mbar $_{2}$ partial pressure [i.e., according to (1-Cl)]. preadsorption and variation in oxygen partial pressure do result in profound changes in cerium oxide film and interface structure, which is shown based on LEED and XRD in the next sections.

\section{B. Low-energy electron diffraction}

A distinct difference between the different growth recipes is noticed when comparing the respective LEED patterns. The very faint $(1 \times 1)$ diffraction spots that appear for recipe (1) completely vanish when changing to recipe (5) (images not shown), pointing toward substantial noncrystallinity of the film, probably due to increased silica or silicate formation with increasing oxygen partial pressure. However, an opposite trend is observed for cerium oxide growth on the Cl-terminated silicon surface. For growth recipe (1-Cl), on average only a very faint, threefold $(1 \times 1)$ LEED pattern is noticed, which becomes much stronger for $(5-\mathrm{Cl})$, indicating the growth of an ordered cerium oxide film of cubic symmetry [see Fig. 1(c)] while hexagonal ${ }^{26}$ and monoclinic ${ }^{27}$ phases can be excluded. Thus, in combination with the XPS results presented in the preceding section, which corroborate the presence of $\mathrm{Ce}^{3+}$ for all preparation conditions investigated, we infer that the cerium oxide films grown on silicon are composed of cubic $\mathrm{Ce}_{2} \mathrm{O}_{3}$; i.e., they crystallize in the cubic bixbyite structure. We also point out that an increased oxygen partial pressure seems to reduce surface crystallinity in the case of oxide growth on the bare surface, whereas it appears to be beneficial in the case of oxide deposition on the Cl-passivated surface.

In the following, we test these rather qualitative considerations by quantitative XRD analysis for film thicknesses in the few-nanometer regime (Sec. III C) and by x-ray standing waves for truly ultrathin films with a nominal cerium oxide coverage of about 1-2 nm (Sec. III D).

\section{X-ray reflectivity and diffraction}

Representative XRD data recorded in the vicinity of the specular $\mathrm{Si}(111)$ Bragg reflection of a cerium oxide film, which was grown at a substrate temperature of $500^{\circ} \mathrm{C}$ according to recipe (1) and capped at room temperature by a 30-nm-thick amorphous silicon layer for protection, is shown in Fig. 4(a). In this XRD section, we use surface coordinates to describe reciprocal space, i.e., in-plane lattice vectors in the [112] and $[\overline{1} 2 \overline{1}]$ directions and out-of-plane in the [111] direction with layer spacing periodicity. Furthermore, we reference the scattering vector $(H K L)$ to the dimensions of the primitive Brillouin zone of the Si crystal using the same coordinate system as a reference. For example, in the case of momentum transfer exhibiting only a normal component $Q_{\perp}$, these "reciprocal lattice units" (r. 1. u.) are related to the layer spacing periodicity $d_{\mathrm{Si}}$ by the relation $Q_{\perp}=L\left(2 \pi / d_{\mathrm{Si}}\right)$, yielding the relation $(111)_{\text {bulk }}=(001)_{\text {surface }}$ between the conventional bulk and the introduced surface notation. According to Fig. 4(a), the position of the Bragg peak of the film yields a lattice plane period of $3.28 \AA$, which is about $1.8 \%$ larger than the reported value for the $\mathrm{O}_{x}-\mathrm{Ce}-\mathrm{O}_{x}$ trilayer $^{28}$ (TL) periodicity of the bulk $\mathrm{Ce}_{2} \mathrm{O}_{3}$ cubic bixbyite structure (3.22 $\AA$ ). Additionally, a quantitative analysis of the pronounced fringes in the diffracted intensity yields an average crystalline cerium oxide film thickness of $5.1 \pm 0.1 \mathrm{~nm}$. 
(a)

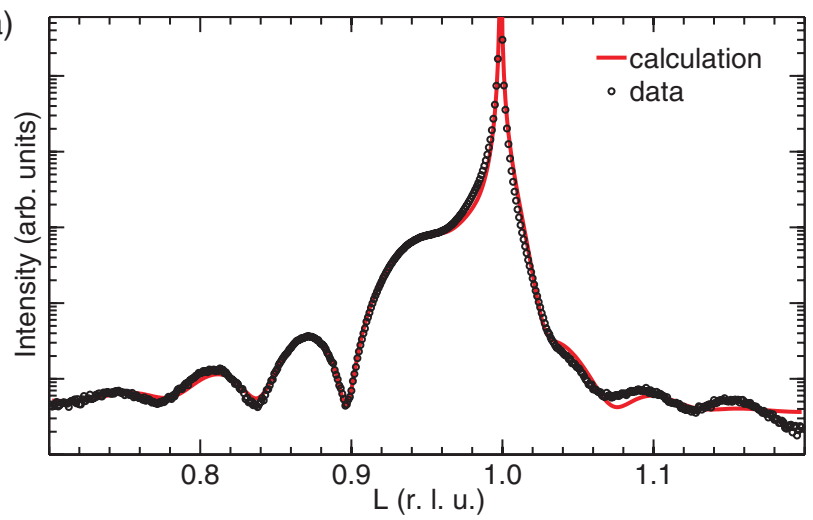

(b)

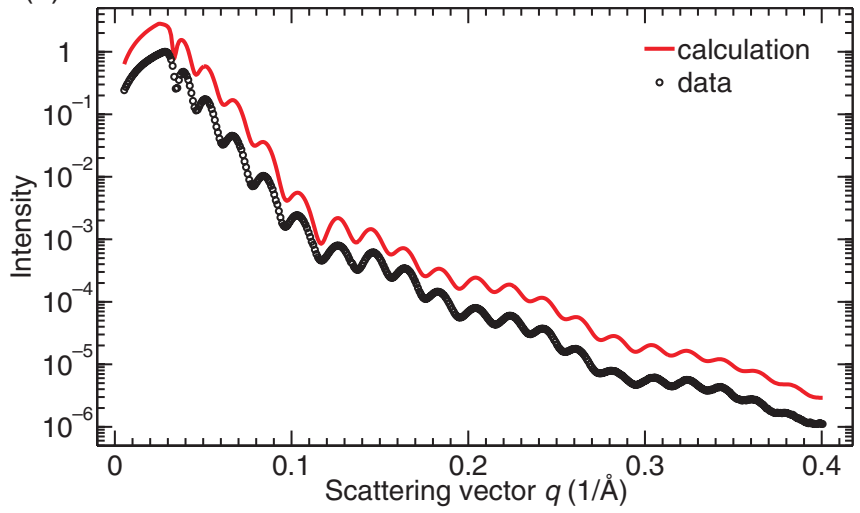

FIG. 4. (Color online) (a) X-ray diffraction and (b) X-ray reflectivity data [open (black) circles] and theoretical fit [solid (red) lines] obtained after cerium oxide growth at $1 \times 10^{-7}$ mbar $\mathrm{O}_{2}$ backfilling and a substrate temperature of $500^{\circ} \mathrm{C}$ on bare $\mathrm{Si}(111)$ and subsequent capping [i.e., following recipe (1-capped)]. In (b), the curves are shown with a small offset, for clarity.

Further information regarding the interface morphology may be derived from the observed asymmetry of the oxide Bragg peak and the overall modulation of the oscillation amplitude. Physically, these characteristics can be related to a phase shift between the diffracted beams of the crystalline overlayer and the substrate, which is attributed to the existence of an amorphous buffer layer. ${ }^{29}$ A quantitative XRD analysis according to Ref. 29 based on the kinematic theory of $\mathrm{x}$-ray diffraction [represented by the solid line in Fig. 4(a)], which also includes the features discussed above, yields a thickness of $1.7 \mathrm{~nm}$ for the interface layer that originates from Ce-catalyzed silicon oxide $\left(\mathrm{SiO}_{x}\right)$ and silicate ( $\left.\mathrm{Si}-\mathrm{O}-\mathrm{Ce}\right)$ formation. ${ }^{10}$

This structural model is corroborated by the XRR investigations as shown in Fig. 4(b). While the short-period oscillations allow to determine the thickness of the capping layer, the more extended modulations are caused by the thin cerium oxide layer and the interface. The quantitative analysis yields a 1.4-nm-thick interface layer, which is in very good agreement with XRD. In the following, we show that the lower limit of a few nanometers for the silicate thickness may be pushed down to the $\mathrm{ML}$ range by introducing an atomic layer of $\mathrm{Cl}$ at the interface.

The $(00 L)$ XRD data obtained in the vicinity of $L=1.0$ for recipe (1-Cl) are shown in Fig. 5(a). From the position of the overlayer Bragg peak, a contracted TL periodicity of
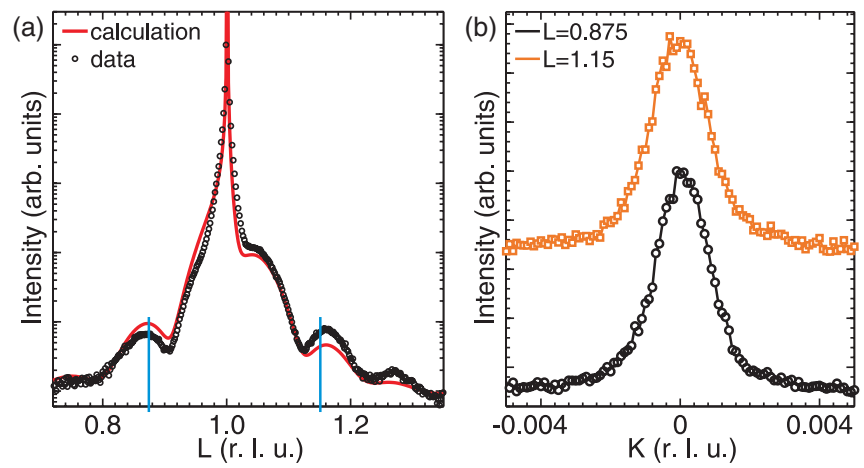

FIG. 5. (Color online) XRD data obtained after cerium oxide growth at $1 \times 10^{-7} \mathrm{mbar}_{2}$ backfilling and a substrate temperature of $500^{\circ} \mathrm{C}$ on $\mathrm{Cl} / \mathrm{Si}(111)$ and subsequent exposure to air [i.e., following recipe (1-Cl-uncapped)]: (a) $(00 L)$-CTR, (b) $(0 K L)$ scans at two different $L$ values used to determine the average grain size. The (red) solid line in (a) represents a calculation following kinematic diffraction theory.

$3.13 \AA$ is deduced, which is close to the expected value of $3.12 \AA$ for $\mathrm{CeO}_{2}$. The apparent discrepancy with the XPS data presented in Sec. III A is reconciled when this change in oxidation state is attributed to postoxidation of the film at ambient conditions because in this case no silicon capping was applied, a hypothesis that was verified by XPS after the XRD measurement (data not shown). Theoretical simulation yields a crystalline film thickness of $2.5 \mathrm{~nm}$. But what is most striking is the result for the amorphous interface layer, which amounts to only $0.4 \mathrm{~nm}$ and is thus on the order of a single trilayer, proving that, despite postoxidation, it is indeed possible to virtually suppress silicate formation.

We now move on to a more detailed investigation of the crystalline properties of the oxidized cerium oxide film. Figure 5(b) shows two in-plane cuts through reciprocal space at the $L$ values of 0.875 and 1.15 r.l.u., i.e., at the first-order maxima of the oscillation determined from the corresponding $(00 L)$ crystal truncation rod (CTR) depicted in Fig. 5(a). Based on a Lorentzian fit of the peak profile in the $K$ direction, a lateral grain size of $(220 \pm 20) \mathrm{nm}$ is inferred. Again, this result demonstrates the relatively high degree of crystallinity of the oxide film, especially considering the postoxidation process.

In Fig. 6, a cut along the $(01 L)$ CTR, i.e., at a fixed, nonvanishing in-plane $(H K)$ component of the scattering vector, is displayed, thereby effectively probing the stacking sequence of the film at the interface. The strong intensity observed at $L \approx \frac{1}{3}$ and $L \approx \frac{4}{3}$ (both denoted $\mathrm{B}$ in the figure) correspond to the (1111) and (202) Bragg peaks (bulk coordinates), respectively, for $B$-oriented cubic cerium oxide, hence indicating the presence of a stacking fault at the interface, which has already been observed for ceria growth on H-passivated $\mathrm{Si}(111){ }^{30}$ However, the discernible peak at $L \simeq \frac{2}{3}$ suggests the coexistence of domains exhibiting regular, $A$-type stacking and can be associated with the (020) reflection, which is allowed for the fluorite structure $(F m \overline{3} m)$ but forbidden for the diamond structure $(F d \overline{3} m)$. It should be noted that the pronounced modulations of the diffracted intensity in the vicinity of the $\left(01 \frac{1}{3}\right)$ reflection are yet again in line with the presence of a sharp, well-defined interface, 


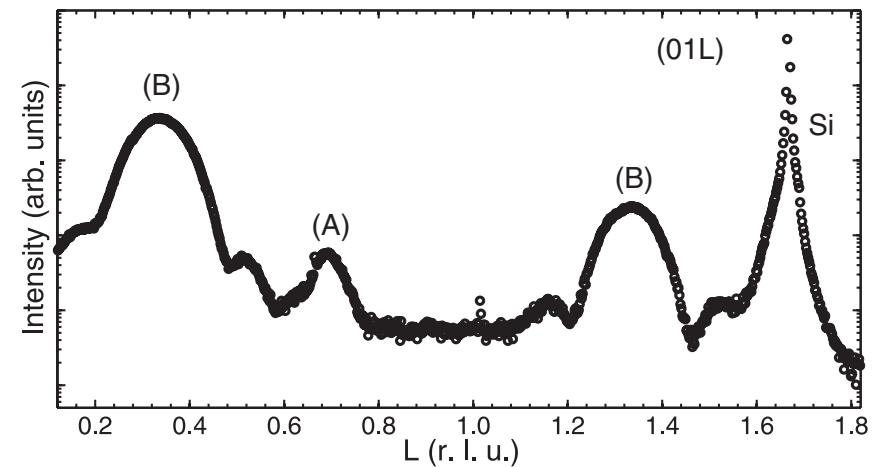

FIG. 6. $\mathrm{Si}(01 L)$ crystal truncation rod data obtained in GIXRD geometry after cerium oxide growth on $\mathrm{Cl} / \mathrm{Si}(111)$ at $1 \times 10^{-7} \mathrm{mbar}$ $\mathrm{O}_{2}$ backfilling and a substrate temperature of $500^{\circ} \mathrm{C}$ and subsequent exposure to air [i.e., following recipe (1-Cl-uncapped)].

corroborating the findings from the analysis of the $(00 L)$ CTR data.

In the following, we move on to the investigation of few-monolayer thin films using XSW to characterize the film crystallinity and the interface at the initial growth stage as well as the role of chlorine in the growth process, which cannot be deduced from the diffraction data presented above.

\section{X-ray standing waves}

For clarity, this section has been subdivided into three parts. In the first section, we introduce the XSW analysis for epitaxial films using the bixbyite structure as an example. In the second section, we present our experimental results for the ultrathin cerium oxide films grown for the four recipes discussed in Sec. III A. Finally, in the last section we investigate the function of chlorine preadsorption in the growth process.

\section{Theoretical XSW analysis of ultrathin bixbyite films}

In this section we present the more fundamental aspects of our XSW analysis of epitaxial $\mathrm{Ce}_{2} \mathrm{O}_{3}$ bixbyite films. Here, we use the conventional bulk notation for reciprocal space. Further information regarding the determination of crystalline film structures using XSW can be found, e.g., in the review article by Zegenhagen. ${ }^{31}$

In an XSW experiment, the Bragg reflectivity and the intensity of an element-specific inelastic signal, e.g., x-ray fluorescence, are simultaneously monitored. By fitting the reflectivity and the yield of the secondary signal according to the dynamical theory of X-ray diffraction, ${ }^{32,33} \mathrm{XSW}$ allows to selectively determine the modulus $f_{c}^{h k l}$ ("coherent fraction") and phase $\Phi_{c}^{h k l}$ ("coherent position") of the $(h k l)$ Fourier component of the spatial distribution function of the contributing atoms. ${ }^{34}$ In the following, we discuss the interpretation of the Fourier coefficients $\mathcal{F}^{h k l}=f_{c}^{h k l} \exp \left(2 \pi i \Phi_{c}^{h k l}\right)$ for the special case of $h=k=l=1$ and the cubic bixbyite film geometry.

The ideal atomic structure of a single layer of bixbyite $\mathrm{Ce}_{2} \mathrm{O}_{3}(111)$ on $\mathrm{Si}(111)$ is shown in Fig. 7. Contrary to the case of $\mathrm{CeO}_{2}$, which crystallizes in the cubic fluorite structure with one well-defined $\mathrm{Ce}$ position, the $\mathrm{Ce}$ atoms occupy symmetrically inequivalent sites with respect to the (111) diffraction planes; hence, it is only the average Ce distance

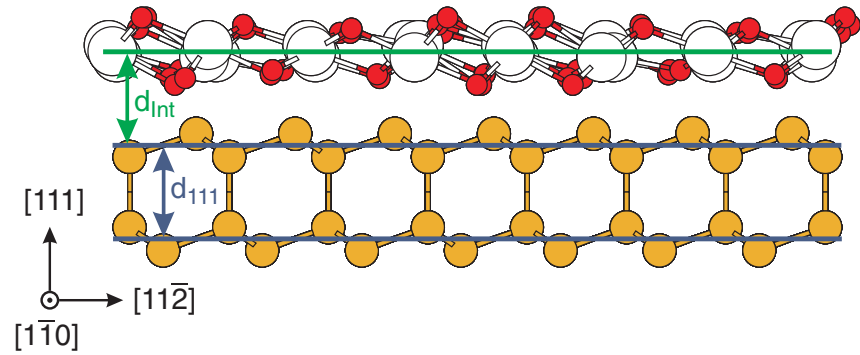

FIG. 7. (Color online) Structural model for a single $\mathrm{Ce}_{2} \mathrm{O}_{3}$ bixbyite trilayer on $\mathrm{Si}(111)$. The parameter $d_{\text {int }}$, which is accessible (modulo the Si layer spacing $d_{111}$ ) in XSW experiments in (111) Bragg reflection, denotes the average distance between the $\mathrm{Ce}$ atoms of the trilayer closest to the substrate and the outermost Si bilayer.

to the underlying bulk $d_{\text {int }}$ that is represented by the measured coherent position $\Phi_{\mathrm{Ce}}^{111}=\left(d_{\text {int }} / d^{h k l}\right) \bmod 1$. Furthermore, this positional averaging leads to an accompanying maximum coherent fraction $f_{\mathrm{Ce}, \max }^{111}=0.945$ for the unstrained bixbyite geometry, i.e., using the same bond lengths as in the bulk crystal. This value is considerably smaller than unity, which is the value that would be expected for single-site $\mathrm{CeO}_{2}$.

A further decrease of $f_{c}^{111}$ arises from measurements at finite temperature $T$ (here $300 \mathrm{~K}$ ), resulting in thermal vibrations of the investigated atoms. Although generally difficult to quantify theoretically or experimentally, ${ }^{31}$ this incoherent motion can be expected to give rise to an anisotropic Debye-Waller factor $D^{h k l}(T)$, which typically is on the order of about 0.95 for moderately heavy atoms. In the isotropic limit, this factor is given by $D(T)=\exp \left(-2 B \sin ^{2} \Theta_{B} / \lambda^{2}\right)$ with the Bragg angle $\Theta_{B}$, the wavelength $\lambda$ of the incident $\mathrm{X}$ rays, and the atomic displacement factor $B=8 \pi^{2}\left\langle u^{2}\right\rangle$, which can be calculated from the atomic vibration amplitude $u$. As a consequence, the Debye-Waller factor limits the upper boundary of $f_{c}$ to $D^{h k l} f_{\mathrm{Ce}, \max }^{111}$ even in the case of perfect structural order. Defects, e.g., at the interface between the film and the substrate, may be represented by an additional factor $0 \leqslant U \leqslant 1$, which describes the percentage of randomly distributed $\mathrm{Ce}$ atoms with respect to the substrate. ${ }^{31}$ The measured coherent fraction is then given by the product $(1-U) D^{h k l} f_{\text {Ce, } \max }^{111}$.

In the case of epitaxial growth on non-lattice-matched substrates, the mismatch in lattice constants will give rise to a shift in the coherent position as well as a decrease of the coherent fraction, scaling with the number of layers. For example, for a 5-TL-thick $\mathrm{Ce}_{2} \mathrm{O}_{3}$ film exhibiting a bulklike layer periodicity and structure and neglecting both thermal vibrations and partial disorder, the ideal coherent fraction is slightly reduced to 0.917 simply by geometry, as compared to 0.945 for the single trilayer. Moreover, compressive lateral strain typically concurs with an even further expanded vertical lattice constant of the film, which will result in a further decrease of the coherent fraction. This situation is schematically illustrated in Fig. 8 for the case of three $\mathrm{Ce}_{2} \mathrm{O}_{3}$ trilayers, where an increasing distance between the trilayer center (average vertical Ce position) and the (111) diffraction planes with an increasing number of trilayers is readily observed.

It is instructive to visualize the result of the numerical XSW simulation in the Gaussian (complex) plane, in which 


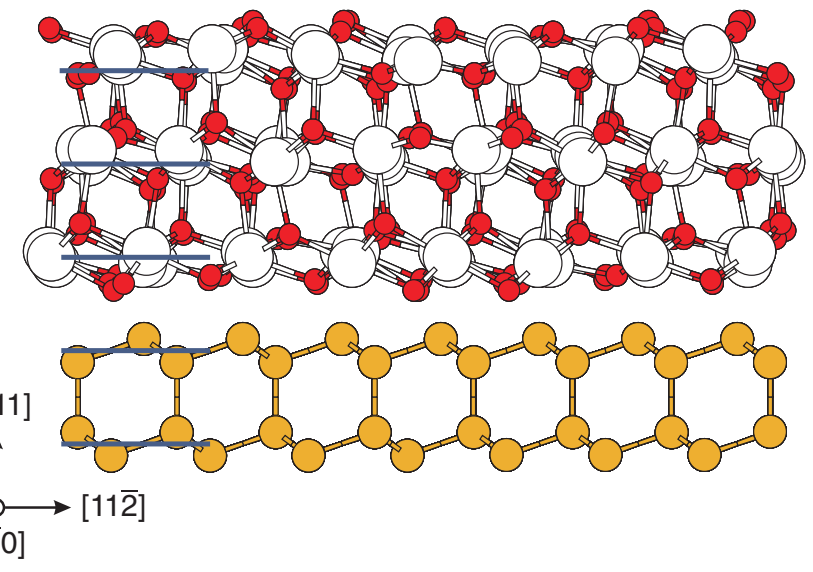

FIG. 8. (Color online) Structural model for three trilayers of cubic (bixbyite) $\mathrm{Ce}_{2} \mathrm{O}_{3}$ compressively strained on $\mathrm{Si}(111)$, assuming volume conservation of the film. The (111) diffraction planes of the substrate-generated standing-wave field are indicated on the left, demonstrating the expected decrease in the Ce coherent fraction with film thickness and vertical lattice mismatch, respectively.

the Fourier coefficients are indicated in the so-called Argand diagram using polar coordinates $\left(f_{\mathrm{Ce}}^{111}, \Phi_{\mathrm{Ce}}^{111}\right)$. In this simulation, whose results are presented in Fig. 9, we considered the scenario of up to eight perfectly ordered bixbyite trilayers of $\mathrm{Ce}_{2} \mathrm{O}_{3}$ (111) epitaxially grown on $\mathrm{Si}(111)$, neglecting DebyeWaller contributions and assuming a strain release with respect to the $\mathrm{Ce}_{2} \mathrm{O}_{3}$ (111) bulk layer periodicity of $2 \%$ [Fig. 9(a)] and $4 \%$ [Fig. 9(b)], respectively. Especially in the latter case, it is clearly visible that the coherent fraction is decreased stepwise with increasing number of trilayers while the layerwise shift $\Delta \Phi$ in the coherent position leads to a distinct rotation around the origin. Comparing the cases in Figs. 9(a) and 9(b), we note that double strain release does not result in a doubled layerwise shift $\Delta \Phi$ due to the already different bulk lattice constants at zero strain release, effectively giving rise to an offset $\Delta \Phi_{0}$ and yielding a total layerwise shift depending on strain release $z$ as $\Delta \Phi=\Delta \Phi_{0}+\Delta \Phi_{z}$. Therefore, as a general result we may conclude that if the number of layers is known, e.g., due to the use of a calibrated deposition source, and if the film is assumed to be perfectly ordered and homogeneous, then a comparison with the experimental XSW result will impose a distinct upper limit on the amount of vertical strain release since the simulated fraction may not be smaller than the experimental one. Furthermore, we may also analyze the coherent position and, starting from the experimentally determined position and using the projected layerwise shift in the reverse direction, deduce the average position of the $\mathrm{Ce}$ atoms in the lowest trilayer with respect to the underlying silicon crystal; i.e., we may determine the interface distance $d_{\text {int }}$ introduced earlier (cf. Fig. 7), albeit only modulo the silicon layer spacing $d_{111}$ (3.14 $\AA$ ). Hence, we may also gain information on the interface thickness from XSW.

While the bixbyite film geometry leads to a maximal coherent fraction for the cation site in the idealized case of vanishing lattice mismatch and zero strain release between the oxide film and the substrate, the inverse characteristic is noted for the anion site. The periodicity of the oxygen sublattice planes in the (111) direction induces a relative
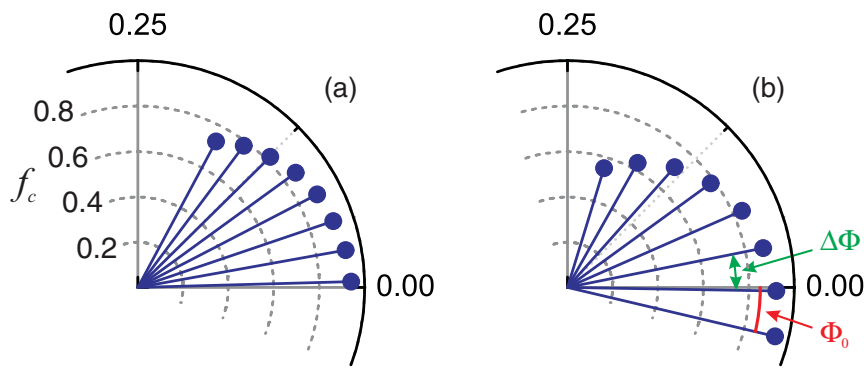

FIG. 9. (Color online) Argand diagrams showing the simulated (111) XSW results depending on relative vertical lattice mismatch and the number of oxygen-metal-oxygen trilayers of $\mathrm{Ce}_{2} \mathrm{O}_{3}(111)$ grown on $\mathrm{Si}(111)$, which are assumed to adopt the bixbyite structure exhibiting a tetragonal distortion of (a) $2 \%$ and (b) $4 \%$ along [111] with respect to the bulk. $\Delta \Phi$ denotes the layerwise shift in coherent position for an increasing number of trilayers, while $\Phi_{0}$ marks the coherent position of the $\mathrm{Ce}$ atoms in the lowest (first) trilayer, which can be deduced from the experimental result.

phase shift of $\pi$ for adjacent planes, i.e., $\Delta \Phi=0.5$, which results in the cancellation of the individual contributions of inelastic signals from consecutive sublattice planes to the total XSW yield. Also in the more realistic case of finite lattice mismatch and strain release up to a few percent, the coherent fraction for an oxygen-specific signal will remain close to zero $\left(f_{\mathrm{O}, \mathrm{max}}^{111} \lesssim 0.1\right)$, impeding a structural analysis of the oxygen sites within the film.

\section{Experimental XSW results for oxide growth on Cl-passivated and unpassivated substrates}

XSW data were recorded in $\mathrm{Si}(111)$ Bragg reflection at a photon energy of $5.9 \mathrm{keV}$ using $\mathrm{Ce} L \alpha$ fluorescence as a secondary signal. In Fig. 10(a), the reflectivity and yields are shown for samples grown at an oxygen partial pressure of $1 \times 10^{-7}$ mbar with and without $\mathrm{Cl}$ passivation. For the conditions $(1-\mathrm{Cl})$ and (1), we obtain coherent fractions $f_{\mathrm{Ce}}^{111}$ of 0.40 and 0.49 , respectively, while the coherent position $\Phi_{\mathrm{Ce}}^{111}=1.00$ is the same for both recipes. Because of the same nominal film thickness, these Fourier coefficients indicate a slightly decreased crystalline order of the $\mathrm{Ce}$ atoms for (1-Cl).

A striking difference in the XSW results is noticed when the oxygen pressure is increased to $5 \times 10^{-7}$ mbar [Fig. 10(b)]. In this case, we observe a strongly increased coherent fraction of 0.70 for recipe $(5-\mathrm{Cl}$ ) as compared to 0.51 for recipe (5), while the coherent positions turn out to be very similar. However, the increased oxygen pressure does not have quite an impact on the crystallinity of the oxide film prepared without $\mathrm{Cl}$ passivation since (5) essentially leads to the same result as (1) (within the error bar).

For the quantitative XSW simulation of results for recipe (5-Cl), we assumed a laterally fully strained $\mathrm{Ce}_{2} \mathrm{O}_{3}$ film of 5-TL thickness using a trilayer spacing of $3.32 \AA$ as determined from continuum elasticity theory based on experimental elastic constants for $\mathrm{CeO}_{2}$ (Ref. 35), yielding a theoretical coherent fraction of 0.817 . Furthermore, thermal vibrations were considered by assuming $D^{111}=0.95$, which is somewhat less than the isotropic Debye-Waller factor $D\left(\mathrm{CeO}_{2}\right)=0.977$ calculated for $\mathrm{CeO}_{2}$ based on an atomic 

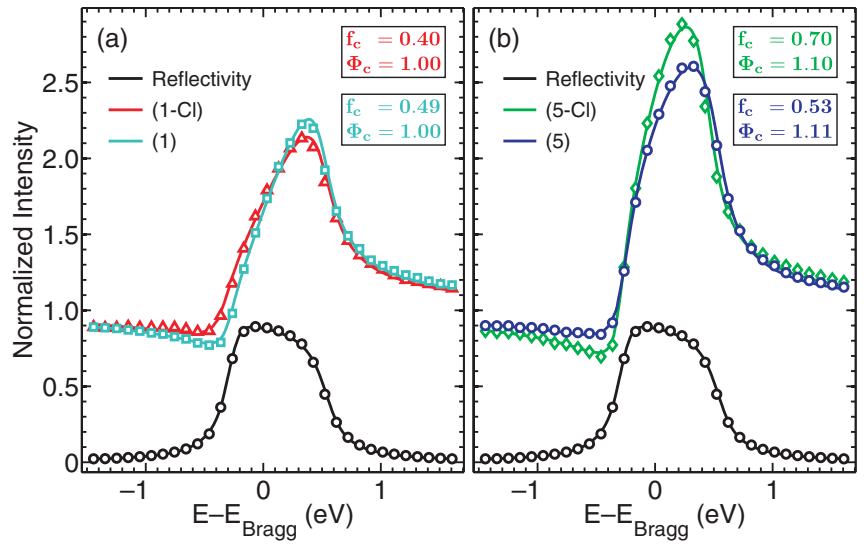

FIG. 10. (Color online) XSW data (open symbols) and theoretical fit according to the dynamical theory of $\mathrm{x}$-ray diffraction (solid lines) using Ce $L \alpha$ fluorescence obtained in $\mathrm{Si}(111)$ Bragg reflection for $(4.9 \pm 0.5)$-TL-thin cerium oxide films on (a) $\mathrm{Si}(111)$ and (b) Cl-passivated $\mathrm{Si}(111)$ for growth at oxygen partial pressures of $1 \times 10^{-7} \operatorname{mbar}[(1),(1-\mathrm{Cl})]$ and $5 \times 10^{-7} \operatorname{mbar}[(5),(5-\mathrm{Cl})]$, respectively.

displacement parameter of $B=0.45 \AA^{2}$ at room temperature as determined by neutron diffraction. ${ }^{36}$ Perfect agreement with the experimental value of 0.70 can be obtained by taking into account modified $\mathrm{Ce}$ sites, yielding a disordered fraction of about $U=0.1$, only weakly depending on the exact value of $D^{111}\left[U=0.12\right.$ if $D^{111}=D\left(\mathrm{CeO}_{2}\right)$ is assumed]. Since this value indicates that fewer than the number of $\mathrm{Ce}$ atoms within a single trilayer are affected, we may speculate that mostly the $\mathrm{Ce}$ atoms at the interface are removed from their ideal bonding site. However, XSW modeling for sample (5) in an analogous fashion shows that about one-third (34\%) of the Ce atoms appear to be disordered, which, in a similar picture, corresponds to the equivalent of about 1.7 trilayers. This interpretation indicates that more than just the direct interface between the cerium oxide film and the underlying substrate is affected, which therefore also points toward incoherent incorporation of $\mathrm{Ce}$ atoms within the film. Hence, together with the XRD analysis from Sec. III C, the XSW simulation clearly proves both the superior crystalline quality of the cerium oxide film as well as of the oxide-semiconductor interface obtained for recipe (5-Cl).

The coherent position $\Phi_{\mathrm{Ce}}^{111}$ amounts to 1.10 for recipe (5-Cl). Based on the same assumptions as presented in the analysis of the coherent fraction, we may deduce the values for the coherent position of the first trilayer to $\Phi_{0}=0.98$ and the interface distance to about $d_{\text {int }}=3.1 \AA$. Although not prepared at the same conditions, this value is compatible with the interface thickness determined from XRD for a thicker, uncapped film $(0.4 \mathrm{~nm})$ grown according to $(1-\mathrm{Cl})$.

\section{The effect of $\mathrm{Cl}$ passivation}

Lastly, we analyze the role of the chlorine atoms in the growth process using the $\mathrm{Si}(111) \mathrm{XSW}$ data (Fig. 11) with $\mathrm{Cl} K \alpha$ fluorescence as a secondary signal recorded before [Fig. 11(a)] and after [Fig. 11(b)] oxide growth. For the $\mathrm{Cl} / \mathrm{Si}(111)-(1 \times 1)$ template, using a photon energy of $3.35 \mathrm{keV}$ for an increased photoabsorption cross section, the

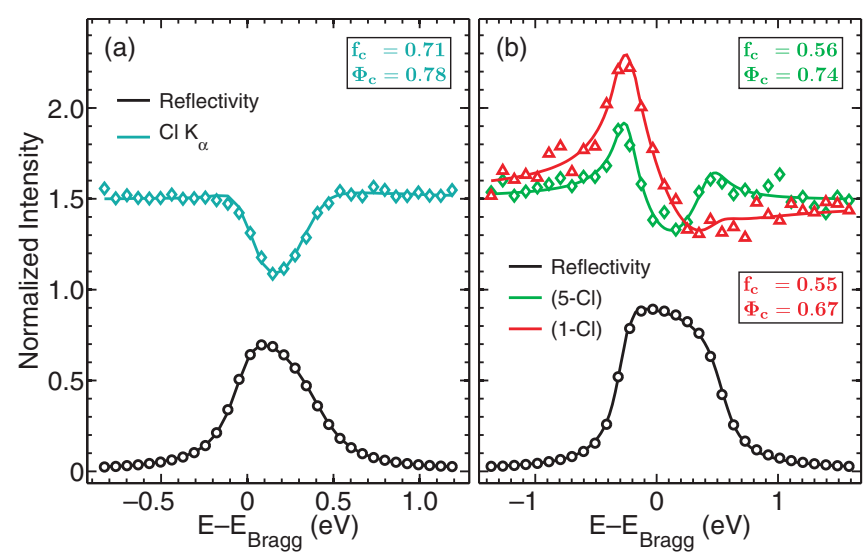

FIG. 11. (Color online) XSW data (data points) and theoretical fit according to the dynamical theory of x-ray diffraction (solid lines) using $\mathrm{Cl} K \alpha$ fluorescence obtained in $\mathrm{Si}(111)$ Bragg reflection for (a) $\mathrm{Cl} / \mathrm{Si}(111)-(1 \times 1)$ and (b) for $(4.9 \pm 0.5)$-TL-thin $\mathrm{Ce}_{2} \mathrm{O}_{3}(111)$ films grown on $\mathrm{Cl}$-passivated $\mathrm{Si}(111)$ at oxygen partial pressures of $1 \times 10^{-7} \mathrm{mbar}(1-\mathrm{Cl})$ and $5 \times 10^{-7} \mathrm{mbar}(5-\mathrm{Cl})$, respectively. All yields are shifted upward by 0.5 units, for clarity.

measured coherent fraction $f_{\mathrm{Cl}}^{111}=0.71$ and coherent position $\Phi_{\mathrm{Cl}}^{111}=0.78$ pinpoint the well-known on-top adsorption site. ${ }^{20,21}$ After cerium oxide growth, we note a different behavior of the $\mathrm{Cl}$ atoms depending on pressure: For preparation according to $(1-\mathrm{Cl})$, both the coherent fraction $(0.55)$ and, especially, the coherent position (0.67) are considerably changed. Hence, we conclude that a relatively large number of $\mathrm{Cl}$ atoms change their adsorption sites upon cerium oxide deposition, rendering the $\mathrm{Cl}$ termination unstable at apparently oxygen-deficient conditions, potentially also favoring cerium oxychloride $(\mathrm{CeOCl})$ formation. This situation, however, is significantly altered at more oxidizing conditions $[(5-\mathrm{Cl})]$. In this case, the coherent positions determined for the $\mathrm{Cl} / \mathrm{Si}(111)$ $(1 \times 1)$ template [0.78, Fig. 11(a)] and after subsequent oxide growth (0.74) are very similar (almost within the error bar of about 0.01), suggesting a more effective passivation of the $\mathrm{Si}(111)$ substrate surface at elevated $\mathrm{O}_{2}$ pressure with respect to oxidation or silicate formation, apparently resulting in an improved crystalline order of the ultrathin cerium oxide film. The slight deviation of the coherent position from the template value after oxide growth indicates that the majority of the $\mathrm{Cl}$ atoms most probably reside at the interface, but possibly in a slightly changed atomic bonding configuration. The decrease in coherent fraction from 0.71 to 0.56 , however, might also be due to a small portion of $\mathrm{Cl}$ atoms that still got stripped off the interface on-top site and could be quite randomly incorporated into the film, filling the randomly distributed oxygen vacancies, analogous to the behavior of $\mathrm{Cl}$ dopant atoms in ceria growth on metal substrates. ${ }^{37}$ Because of the out-of-phase condition for the averaged oxygen position in the bixbyite film with respect to the (111) diffraction planes (cf. Sec. III D1), such a $\mathrm{Cl}$ distribution manifests itself in a reduced overall coherent fraction at an essentially unchanged coherent position.

The notion of $\mathrm{Cl}$ atoms mostly residing at the interface following cerium oxide growth is consistent with $\mathrm{Cl} 1 s$ XPS data recorded at a photon energy of $3.35 \mathrm{keV}$ before and after growth of a 10-nm-thick oxide film (see Fig. 12). In the figure, 


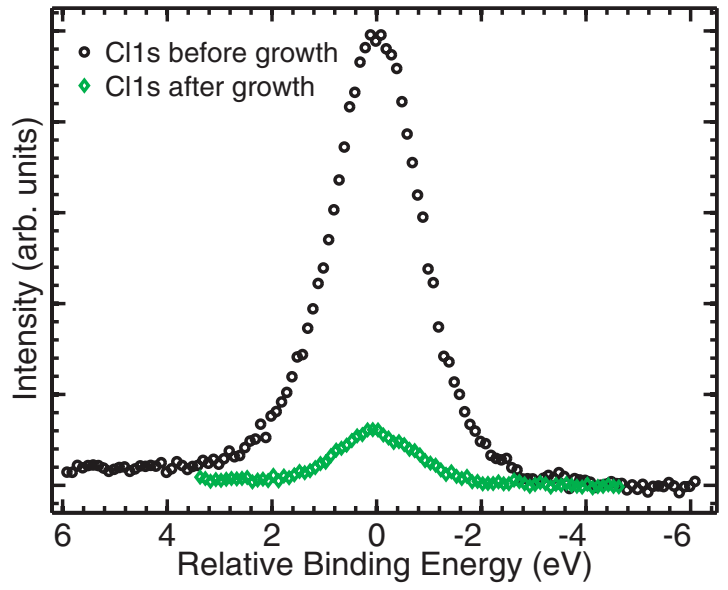

FIG. 12. (Color online) $\mathrm{Cl} 1 s$ XPS data obtained (a) before and (b) after growth of a 10 -nm-thick cerium oxide film at $500^{\circ} \mathrm{C}$ substrate temperature and $5 \times 10^{-7}$ mbar $_{2}$ partial pressure [i.e., according to recipe $(5-\mathrm{Cl})]$.

a strong decrease in photoelectron intensity by one order of magnitude is observed, suggesting that up to about $90 \%$ of the $\mathrm{Cl}$ atoms remain at the interface to the substrate and thus invisible to XPS at these conditions since at a kinetic energy of about $530 \mathrm{eV}$ the photoelectron escape depth is much smaller than the thickness of the film. We also note that the finding of $\mathrm{Cl}$ in the near-surface region supports the idea of partial $\mathrm{Cl}$ surface segregation, while extended oxychloride formation in the film is rendered unlikely.

Together with XPS, the XSW results suggest the following scenario: At too-low oxygen pressure, the Ce deposition rate is higher than the oxygen supply that is necessary for effective Ce oxidation, hence leading to partial cerium silicate or oxychloride formation. This interpretation would also be compatible with the sole existence of $\mathrm{Ce}^{3+}$ ions as determined by XPS. At higher $\mathrm{O}_{2}$ backfilling, however, the abundant oxygen molecules may readily be dissociated and subsequently bound by the metallic, highly reactive $\mathrm{Ce}$, concomitant with the formation of a highly ordered cerium oxide layer, while the silicon substrate remains mostly terminated by chlorine, which protects the silicon layer from direct oxidation. Since all $\mathrm{Ce}$ is oxidized, the oxide layer will in turn stabilize the $\mathrm{Cl}$ termination at the interface. Even in the latter case, the presence of $\mathrm{Cl}$ at the surface may cause a reduced reactivity of $\mathrm{Ce}^{3+}$ ions for further oxidation, ${ }^{38}$ which prevents the growth of a fully oxidized $\mathrm{CeO}_{2}$ film at conditions where $\mathrm{Ce}^{4+}$ ions are readily formed at, e.g., transition-metal surfaces. ${ }^{39}$

\section{CONCLUSION}

Using a multiscale characterization approach we have demonstrated that chlorine passivation is an effective means to almost completely suppress silicate formation in cerium oxide growth on $\mathrm{Si}(111)$ surfaces by reactive deposition of metallic Ce under oxygen backfilling, resulting in the formation of a continuous cubic $\mathrm{Ce}_{2} \mathrm{O}_{3}(111)$ layer. Upon exposure to ambient conditions, the films are converted to a fully oxidized $\mathrm{CeO}_{2}(111)$ fluorite structure exhibiting B-type stacking and an average lateral grain size of about $220 \mathrm{~nm}$. However, the resulting interface layer is shown to remain truly ultrathin, i.e., on the order of about a single trilayer, even after postoxidation.

Furthermore, we find that, depending on the oxygen partial pressure during epitaxy, the $\mathrm{Cl}$ atoms show a tendency toward site exchange (low-pressure regime) or tend to remain at the on-top adsorption sites (elevated-pressure regime), concomitantly stabilizing the interface between the oxide film and the silicon bulk. These findings are expected to have significant implications for the whole class of rare-earth oxides ${ }^{40}$ and their epitaxy on silicon and might eventually pave the road for the successful realization of future high- $k$ dielectrics on silicon surfaces exhibiting sharp, atomically controlled interfaces.

\section{ACKNOWLEDGMENTS}

The authors are indebted to Jürgen Lauckner (University of Bremen) for his invaluable technical support. Portions of this research were carried out at the light source DORIS III at DESY. DESY is a member of the Helmholtz Association (HGF). We would like to thank D. Novikov for assistance in using beam lines BW1 and BW2.

\footnotetext{
*flege@ifp.uni-bremen.de

${ }^{1}$ H. Huff and D. Gilmer, (eds.), High Dielectric Constant Materials: VLSI MOSFET Applications, Springer Series in Advanced Microelectronics (Springer, Berlin, 2004).

${ }^{2}$ Catalysis by Ceria Related Materials, edited by A. Trovaelli (Imperial College Press, London, 2001).

${ }^{3}$ K. J. Hubbard and D. G. Schlom, J. Mater. Res. 11, 2757 (1996).

${ }^{4}$ H. J. Osten, E. Bugiel, O. Kirfel, M. Czernohorsky, and A. Fissel, J. Cryst. Growth 278, 18 (2005).

${ }^{5}$ T. Inoue, Y. Yamamoto, S. Koyama, S. Suzuki, and Y. Ueda, Appl. Phys. Lett. 56, 1332 (1990).

${ }^{6}$ Y. Nishikawa, T. Yamaguchi, M. Yoshiki, H. Satake, and N. Fukushima, Appl. Phys. Lett. 81, 4386 (2002).

${ }^{7}$ Y. Nishikawa, N. Fukushima, N. Yasuda, K. Nakayama, and S. Ikegawa, Jpn. J. Appl. Phys. 41, 2480 (2002).
}

${ }^{8}$ T. Inoue, T. Ohsuna, L. Luo, X. D. Wu, C. J. Maggiore, Y. Yamamoto, Y. Sakurai, and J. H. Chang, Appl. Phys. Lett. 59, 3604 (1991).

${ }^{9}$ H. Nagata, M. Yoshimoto, H. Koinuma, E. Min, and N. Haga, J. Cryst. Growth 123, 1 (1992).

${ }^{10}$ F. U. Hillebrecht, M. Ronay, D. Rieger, and F. J. Himpsel, Phys. Rev. B 34, 5377 (1986).

${ }^{11}$ T. Chikyow, S. M. Bedair, L. Tye, and N. A. El-Masry, Appl. Phys. Lett. 65, 1030 (1994).

${ }^{12}$ E. J. Preisler, O. J. Marsh, R. A. Beach, and T. C. McGill, J. Vac. Sci. Technol. B 19, 1611 (2001).

${ }^{13}$ G.-y. Adachi and N. Imanaka, Chem. Rev. 98, 1479 (1998).

${ }^{14}$ M. Yoshimoto, K. Shimozono, T. Maeda, T. Ohnishi, M. Kumagai, T. Chikyow, O. Ishiyama, M. Shinohara, and H. Koinuma, Jpn. J. Appl. Phys. 34, L688 (1995). 
${ }^{15}$ B. Hirschauer, M. Göthelid, E. Janin, H. Lu, and U. O. Karlsson, Appl. Surf. Sci. 148, 164 (1999).

${ }^{16}$ P. Gupta, V. L. Colvin, and S. M. George, Phys. Rev. B 37, 8234 (1988).

${ }^{17}$ P. H. Citrin, J. E. Rowe, and P. Eisenberger, Phys. Rev. B 28, 2299 (1983).

${ }^{18}$ J. S. Villarrubia and J. J. Boland, Phys. Rev. Lett. 63, 306 (1989).

${ }^{19}$ J. J. Boland and J. S. Villarrubia, Phys. Rev. B 41, 9865 (1990).

${ }^{20}$ J. I. Flege, T. Schmidt, J. Falta, and G. Materlik, Surf. Sci. 507-510, 381 (2002).

${ }^{21}$ J. I. Flege, T. Schmidt, J. Bätjer, M. Çakmak, J. Falta, and G. Materlik, New J. Phys. 7, 208 (2005).

${ }^{22}$ P. Gupta, P. A. Coon, B. G. Koehler, and S. M. George, Surf. Sci. 249, 92 (1991).

${ }^{23}$ N. D. Spencer, P. J. Goddard, P. W. Davies, M. Kitson, and R. M. Lambert, J. Vac. Sci. Technol. A 1, 1554 (1983).

${ }^{24}$ A. Kotani, T. Jo, and J. C. Parlebas, Adv. Phys. 37, 37 (1988).

${ }^{25}$ D. R. Mullins, S. H. Overbury, and D. R. Huntley, Surf. Sci. 409, 307 (1998).

${ }^{26}$ H. Bärnighausen and G. Schiller, J. Less-Common Met. 110, 385 (1985).

${ }^{27}$ D. T. Cromer, J. Phys. Chem. 61, 753 (1957).

${ }^{28}$ This notation reflects the structural similarity of the bixbyite structure $(x=0.75)$ and the cubic fluorite structure $(x=1)$.
${ }^{29}$ T. Weisemoeller, F. Bertram, S. Gevers, C. Deiter, A. Greuling, and J. Wollschläger, Phys. Rev. B 79, 245422 (2009).

${ }^{30}$ M. Furusawa, J. Tashiro, A. Sasaki, K. Nakajima, M. Takakura, T. Chikyow, P. Ahmet, and M. Yoshimoto, Appl. Phys. Lett. 78, 1838 (2001).

${ }^{31}$ J. Zegenhagen, Surf. Sci. Rep. 18, 202 (1993).

${ }^{32} \mathrm{M}$. von Laue, Röntgenstrahl-Interferenzen (Akademische VerlagsGesellschaft, Frankfurt, 1960).

${ }^{33}$ B. W. Batterman and H. Cole, Rev. Mod. Phys. 36, 681 (1964).

${ }^{34}$ J. Zegenhagen, G. Materlik, and W. Uelhoff, J. X-Ray Sci. Technol. 2, 214 (1990).

${ }^{35}$ A. Nakajima, A. Yoshihara, and M. Ishigame, Phys. Rev. B 50, 13297 (1994).

${ }^{36}$ M. Yashima, D. Ishimura, Y. Yamaguchi, K. Ohoyama, and K. Kawachi, Chem. Phys. Lett. 372, 784 (2003).

${ }^{37}$ F. Fajardie, J.-F. Tempere, J.-M. Manoli, G. Djega-Mariadassou, and G. Blanchard, J. Chem. Soc., Faraday Trans. 94, 3727 (1998).

${ }^{38}$ J. Soria, A. Martínez-Arias, J. M. Coronado, and J. C. Conesa, Top. Catal. 11/12, 205 (2000).

${ }^{39}$ D. R. Mullins, P. V. Radulovic, and S. H. Overbury, Surf. Sci. 429, 186 (1999).

${ }^{40}$ S. Gevers, J. I. Flege, B. Kaemena, D. Bruns, T. Weisemoeller, J. Falta, and J. Wollschläger, Appl. Phys. Lett. 97, 242901 (2010). 